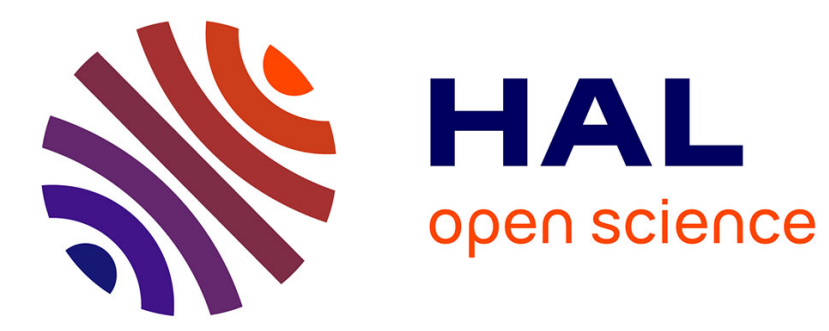

\title{
Collapse of branched polymers
}

\author{
B. Derrida, H.J. Herrmann
}

\section{To cite this version:}

B. Derrida, H.J. Herrmann. Collapse of branched polymers. Journal de Physique, 1983, 44 (12), pp.1365-1376. 10.1051/jphys:0198300440120136500 . jpa-00209724

\section{HAL Id: jpa-00209724 https://hal.science/jpa-00209724}

Submitted on 1 Jan 1983

HAL is a multi-disciplinary open access archive for the deposit and dissemination of scientific research documents, whether they are published or not. The documents may come from teaching and research institutions in France or abroad, or from public or private research centers.
L'archive ouverte pluridisciplinaire HAL, est destinée au dépôt et à la diffusion de documents scientifiques de niveau recherche, publiés ou non, émanant des établissements d'enseignement et de recherche français ou étrangers, des laboratoires publics ou privés. 
Classification

Physics Abstracts

$61.40 \mathrm{~K}-64.70$

\title{
Collapse of branched polymers
}

\author{
B. Derrida and H. J. Herrmann \\ Service de Physique Théorique, CEN-Saclay, 91191 Gif-sur-Yvette Cedex, France
}

(Reçu le 31 mai 1983, accepté le 8 août 1983)

\begin{abstract}
Résumé. - En utilisant la méthode de la matrice de transfert, nous effectuons des calculs exacts sur des rubans de largeur finie pour étudier le problème d'un animal avec une interaction attractive entre sites voisins. Nous calculons des quantités thermodynamiques comme la chaleur spécifique, la compressibilité, le facteur de dilatation. Le scaling sur la taille donne une estimation très précise de la ligne critique en utilisant deux largeurs. Avec trois largeurs ou bien les deux valeurs propres les plus grandes de la matrice de transfert, nous présentons deux façons d'obtenir le point tricritique et ses exposants. Nos estimations sont très stables quand la largeur augmente et nous pouvons donner des prédictions assez précises. Enfin, notre modèle peut aussi être interprété comme un gel, dont les paramètres sont la température et la pression, qui présente le phénomène de collapse bien connu expérimentalement.
\end{abstract}

\begin{abstract}
Exact calculations using transfer matrices on finite strips are performed to study the two-dimensional problem of one lattice animal with an attractive nearest neighbour interaction. Thermodynamic quantities such as specific heat, compressibility, thermal expansion are calculated. Finite size scaling with two strips of different widths yields very accurate approximations of the critical line. Using three different strip widths or the two largest eigenvalues of the transfer matrix, we present two ways of obtaining the tricritical point and its exponents. Our estimations are quite stable when we increase the strip width and we can give rather accurate predictions. Lastly our model can also be interpreted as a gel whose parameters are temperature and pressure showing the experimentally known phenomenon of the collapse.
\end{abstract}

\section{Introduction.}

The problem of a polymer chain collapsing at low temperatures due to the competition between excluded volume and attractive interactions of the monomers of the chains and the corresponding tricritical point $\theta$ have been studied for a long time theoretically [1-10, $16,17,38]$ and also experimentally [11-4]. The attractive forces are induced by interactions with the solvent. In a poor solvent, the monomers of the chain avoid contacts with the solvent and the attractive forces are strong enough to make the chain collapse. On the contrary, in a good solvent, the effective interactions are mostly excluded volume ones. The tricritical exponents at the collapse temperature $\theta$ have been determined by the Flory approximation which is generally acknowledged to be rather good [15]. The relationship $[16,17]$ between the collapse temperature $\theta$ and the usual theta region (defined by the vanishing of the second virial coefficient) is well established $[2,18,7]$.
Much less interest has been paid in the literature to the collapse of branched polymers which is the subject of this paper. By branched polymers, we mean polymers that can have any geometrical configuration including branches or loops, i.e. lattice animals [19]. Experimentally, branched polymers are best realized in gels where indeed some time ago a collapse has been found experimentally [20-21]. Theoretically, meanfield type calculations have been performed [22-23] recently. Flory arguments have been applied to obtain the critical and the tricritical exponents of branched polymers $[24,15]$. But since the upper critical dimension $d_{c}=8$ and the upper tricritical dimension $d_{\mathrm{t}}=6$ for branched polymers [25] whereas $d_{\mathrm{c}}=4$ and $d_{\mathrm{t}}=3$ for linear polymers, it is quite possible that the Flory exponents are not as good in the branched case as in the linear case in physical dimensions. It is likely that, also in the branched case, the collapse point coincides with the point where the second virial coefficient vanishes ( $\theta$ point) [26]. 
We investigate two-dimensional branched polymers, a case for which to our knowledge no experiments exist so far but might be possible [27] (for linear polymers two-dimensional experiments have been performed [13-14]). We look at one single cluster of $N$ connected sites, i.e. one lattice site animal, on a square lattice. Each pair of nearest neighbour sites has an additional attractive energy and so the energy of the whole lattice animal is just the number of all pairs of nearest neighbours. As for the collapse of linear chains, we are interested in studying the geometrical and the thermal properties of an animal of $N$ sites as a function of temperature in the thermodynamic limit $N \rightarrow \infty$.

All the thermal properties (free energy, energy, specific heat) of an animal of $N$ sites can be obtained from the knowledge of the number $\Omega(N, B)$ of different configurations of an animal of $N$ sites with $B$ pairs of nearest neighbours. In the thermodynamic limit, it suffices to know the asymptotic behaviour for large $N$ and $B$ of $\Omega(N, B)$. If we introduce the generating function $G(x, T)$ :

$$
G(x, T)=\sum_{N, B} \Omega(N, B) x^{N}[y(T)]^{B}
$$

where $y$ is related to the temperature $T$ by

$$
y(T)=\exp \left(\frac{1}{T}\right)
$$

the thermal properties in the thermodynamic limit are given by the critical point $\tilde{x}(T)$ where the function $G(x, T)$ becomes singular with respect to $x$.

Indeed, if we define the partition function $Z_{N}$ of an animal of $N$ sites by

$$
Z_{N}=\sum_{B} \Omega(N, B)[y(T)]^{B},
$$

equation 1 can be rewritten as

$$
G(x, T)=\sum_{N} Z_{N} x^{N}
$$

Then the free energy $f(T)$ per site in the animal is given by

$$
f(T)=-T \lim _{N \rightarrow \infty} \frac{1}{N} \log Z_{N}=T \log [\tilde{x}(T)] .
$$

Equation 4 defines the quantity $\tilde{x}(T)$. It is easy to see that it is the radius of convergence of the series (3).

We shall see that at the tricritical point $\theta$ the free energy $f(T)$ per site of the animal is singular.

The most important geometrical property is the average size $\left\langle R^{2}\right\rangle$ of the animal. There are several ways of defining $\left\langle R^{2}\right\rangle$ (for example one can take the radius of gyration) and all of them should give the same exponents. For large $N$, at a given temperature, one expects the following critical behaviour of the average size

$$
\left\langle R^{2}\right\rangle \sim N^{2 v} .
$$

At the collapse transition $\theta$, one expects $v$ to change : above $\theta$, the exponent $v$ should take the value of usual lattice animals [28] $(v \simeq 0.64$ in $d=2)$; below $\theta$, the animal is collapsed $(v=1 / d=1 / 2$ here). Exactly at $\theta$, the exponent $v$ takes a value $v_{1}$ that we shall calculate in the present work. A simple quantity which contains the geometrical information is $g_{O R}(x, T)$ defined by

$$
g_{0 R}(x, T)=\sum_{N, B} x^{N}[y(T)]^{B} \omega_{0 R}(N, B)
$$

where $\omega_{0 R}(N, B)$ is the number of different configurations of an animal of $N$ sites and energy $B$ which connects the points 0 and $R$ of the lattice. This additional condition of connectivity is the only difference between definitions (1) and (6). Like in the case of usual selfavoiding walks or usual lattice animals [28], one can show that, if $x<\tilde{x}(T), g_{0 R}$ decreases with $R$ exponentially. This defines a correlation length $\xi(x, T)$

$g_{0 R}(x, T) \sim \exp [-|R| / \xi(x, T)]$ for large $R$.

One can show that $\xi(x, T)$ diverges when $x \rightarrow \tilde{x}(T)$ and the manner in which $\xi(x, T)$ diverges gives the exponent $v$ of equation 5

$$
\xi^{-1}(x, T) \sim|\tilde{x}(T)-x|^{v} .
$$

Thus we see that the thermal and the geometrical properties of one lattice animal in the limit $N \rightarrow \infty$ are completely described by the neighbourhood of the curve $\tilde{x}(T)$. This does not mean that only this region in the $x-T$ plane is of physical interest. A gel is a macromolecule characterized by the property that it spans from one side of the recipient to the other. In the present paper, we shall use strip geometries to do our calculations. For such geometries, the lattice is infinite in only one direction. One can then consider that

$$
\psi=-T \log \left[g_{0 R}(x, T)\right]
$$

is the grand canonical potential of a " weak " gel problem where the gel is constrained to be connected from column 0 to column $R$ of the strip. Parameters at our disposal are the pressure $p$ and the temperature $T$ and therefore it is not surprising if the whole plane $x, T$ has a physical meaning. "Weak " gel [23] means that under the influence of temperature or pressure, the gel can go over to any new configuration. Moreover, we consider here a situation where the gel is constituted by a single big molecule. In experiments on the contrary, gels are often "strong" i.e. go only over to topologically equivalent configurations because the chemical binding energy is usually larger than thermal energies. However, one can expect that our model can be applied to explain experimental features. 
We note that there are other models for gels particularly ones which include the simultaneous presence of finite polymers (see Ref. 39 for several examples).

In section 2, we explain the transfer matrix technique for this problem. In section 3, we present thermodynamic quantities calculated with this technique on strips of finite width. Section 4 is devoted to the $n$ to $n-1$ renormalization which gives approximately the line $\tilde{x}(T)$ in $d=2$ and estimations of the exponent $v$. In section 5 , we determine the tricritical point $\theta$ and its exponents by two different methods, each of them being applied to two different directions of the strips. In section 6 , we evaluate the thermodynamic quantities at the collapse transition $\theta$. Section 7 summarizes our results. An appendix gives technical details and an example of the calculation.

\section{Transfer matrix for branched polymers.}

We calculate exactly the correlation length $\xi_{n}(x, T)$ defined in (7) on a $n \times \infty$ strip by means of the transfer matrix. The method to do so is very similar to the ones which were used for studying other geometrical problems [28, 29, 30]. As usual, the transfer matrix method consists in writing recursion relations between a strip of length $R$ and a strip of length $R+1$. If we consider a lattice animal on a strip which goes from left to right and if we cut the strip at column $R$, the part of the animal at the left of column $R$ realizes a connectivity configuration $C$ of the sites of column $R$ (see the appendix for an example). Giving $C$ is the same as knowing the occupied sites of column $R$ and how these occupied sites are connected with each other by the part of the strip at the left of $R$. Let us define $g_{0 R}(C)$ by

$$
g_{0 R}(C)=\sum_{N, B} \tilde{\omega}_{0 R}(N, B, C) x^{N} y^{B}
$$

where $\widetilde{\omega}_{0 R}(N, B, C)$ is the number of configurations of the left part of the strip with $N$ occupied sites and energy $B$ which connect column 0 to column $R$ and realize $C$ in column $R$. By definition, the transfer matrix $M$ is the recursion relation between the $g_{0 R}(C)$ :

$$
g_{0 R+1}(C)=\sum_{C^{\prime}} M\left(C, C^{\prime}\right) g_{0 R}\left(C^{\prime}\right)
$$

Obviously, the first thing one must know is the size $s$ of the matrix, i.e. the number of different configurations $C$. This is not a trivial task for connectivity problems and except for narrow strips, one needs a computer. We shall discuss a method to do that in the appendix. Once $s$ is known, one calculates $M$ by

$$
M\left(C, C^{\prime}\right)=x^{t(C)} y^{u\left(C, C^{\prime}\right)}
$$

where $t(C)$ is the number of occupied sites of $C$, $u\left(C, C^{\prime}\right)$ is the number of nearest neighbour pairs of occupied sites in $C$ and between $C$ and $C^{\prime}$. The size of the matrix can be strongly reduced by the use of symmetry operations. An example is given in the appendix.

As $M$ does not depend on $R$, one can, once constructed $M$, calculate $g_{0 R}$ for very large $R$ by iterating (11). If $\lambda$ is the largest eigenvalue of $M(\lambda$ is obviously positive since all the elements of $M$ are $\geqslant 0$ ), each $g_{0 R}$ has the following behaviour

$$
g_{0 R}(C) \sim \lambda^{R} \quad \text { for large } R .
$$

This means that for the strip of width $n$, the correlation length $\xi_{n}(x, T)$ is given by

$$
\xi_{n}(x, T)=-(\log \lambda)^{-1} .
$$

One should not be surprised that by (14) one relates the correlation length to the largest eigenvalue of the transfer matrix. This was also the case in other geometrical problems [28]. There is always an additional configuration $C_{0}$ which is empty and which can be left out but would give an extra eigenvalue 1 .

We can always calculate $\lambda$ with the accuracy we want by sufficiently iterating the matrix $M$.

In this paper we will only consider the square lattice but we will define two different types of strips on it : one in the $(1,0)$ direction and one in the $(1,1)$ direction. We call the first one normal and the second one diagonal (Fig. 1). The reason of studying at the same time these two directions is that the results are much more reliable when they are obtained in two different ways. Let us mention that the diagonal strips were already used in the study of directed percolation [31] and directed animals [32].

\section{Calculation of the thermodynamic properties on strips.}

In this section, we want to describe the properties of one animal of $N$ sites, in the limit $N \rightarrow \infty$, when this animal is confined on a strip of finite width $n$. All those properties can be obtained from the knowledge of the largest eigenvalue $\lambda(x, T)$ of the transfer matrix. Moreover, as explained in the introduction since we are interested only in the limit $N \rightarrow \infty$, it is sufficient to know $\lambda(x, T)$ in the neighbourhood of the line $\tilde{x}_{n}(T)$ where the correlation length $\xi_{n}(x, T)$ defined by (14) diverges.

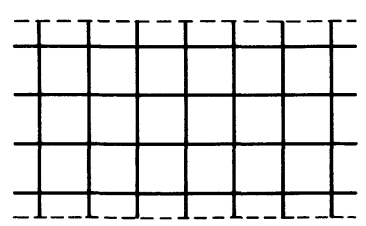

12

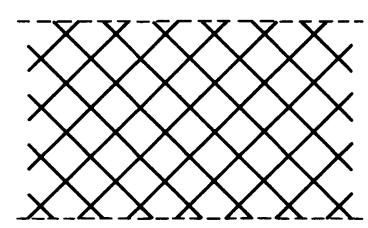

$1 b$
Fig. 1. - (a) A normal strip of width $n=4 ;(b)$ a diagonal strip of width $n=4$. Periodic boundary conditions are fulfilled if one identifies the two dashed lines. 
The eigenvalue $\lambda(x, T)$ or the correlation length $\xi_{n}(x, T)$ are expressed as a function of temperature $T$ and of the parameter $x$ which is conjugate to the number of sites in the animal. It is not very hard to come back to the variable $N$. First, as we saw in (4) of the introduction the free energy $f_{n}(T)$ per site of the animal in the limit $N \rightarrow \infty$ is given by

$$
f_{n}(T)=T \log \left[\tilde{x}_{n}(T)\right]
$$

where $\tilde{x}_{n}(T)$ is the smallest positive value of $x$ for which

$$
\lambda\left[\tilde{x}_{n}(T), T\right]=1 .
$$

Therefore the energy $e_{n}$ and the specific heat $C_{n}$ are given by

$$
e_{n}=-T^{2} \frac{\mathrm{d} \tilde{x}_{n}(T)}{\mathrm{d} T}
$$

and

$$
C_{n}=-2 T \frac{\mathrm{d} \tilde{x}_{n}(T)}{\mathrm{d} T}-T^{2} \frac{\mathrm{d}^{2} \tilde{x}_{n}(T)}{\mathrm{d} T^{2}}
$$

The figure 2 represents $C_{n}$ as a function of temperature for several strip widths.

One can also obtain the geometrical properties of an animal very easily. This was done by Klein [33] in the case of self avoiding walks. We have here exactly the same expression. The average size $R$ of an animal of $N$
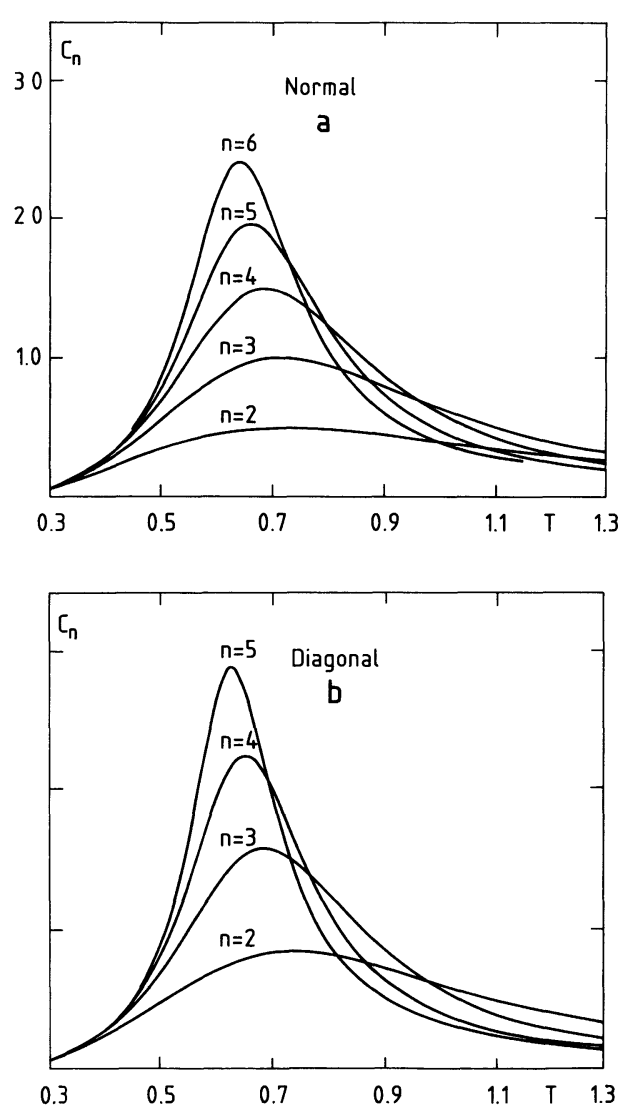

Fig. 2. - Specific heat $C_{n}$ against temperature for different strip widths $n ;(a)$ normal strip direction; (b) diagonal strip direction. sites on a strip of width $n$ is given by

$$
R=N\left[\frac{\partial \log \lambda}{\partial \log x}\left(\tilde{x}_{n}(T), T\right)\right]^{-1} .
$$

Since the strip is a one-dimensional lattice, it is not surprising that $R$ is proportional to $N$. On a strip the exponent $v$ is equal to one at any temperature. From (19), one can calculate the density $\rho_{n}(T)$ of an animal in the limit $N \rightarrow \infty$. Since there is an animal of $N$ sites (with the relation 19 between $R$ and $N$ ) in a rectangle of area $R n$, the density is given by

$$
\rho_{n}=\frac{1}{n} \frac{\partial \log \lambda}{\partial \log x}\left(\tilde{x}_{n}(T), T\right) .
$$

Figure 3 gives $\rho_{n}$ as a function of temperature for different $n$.
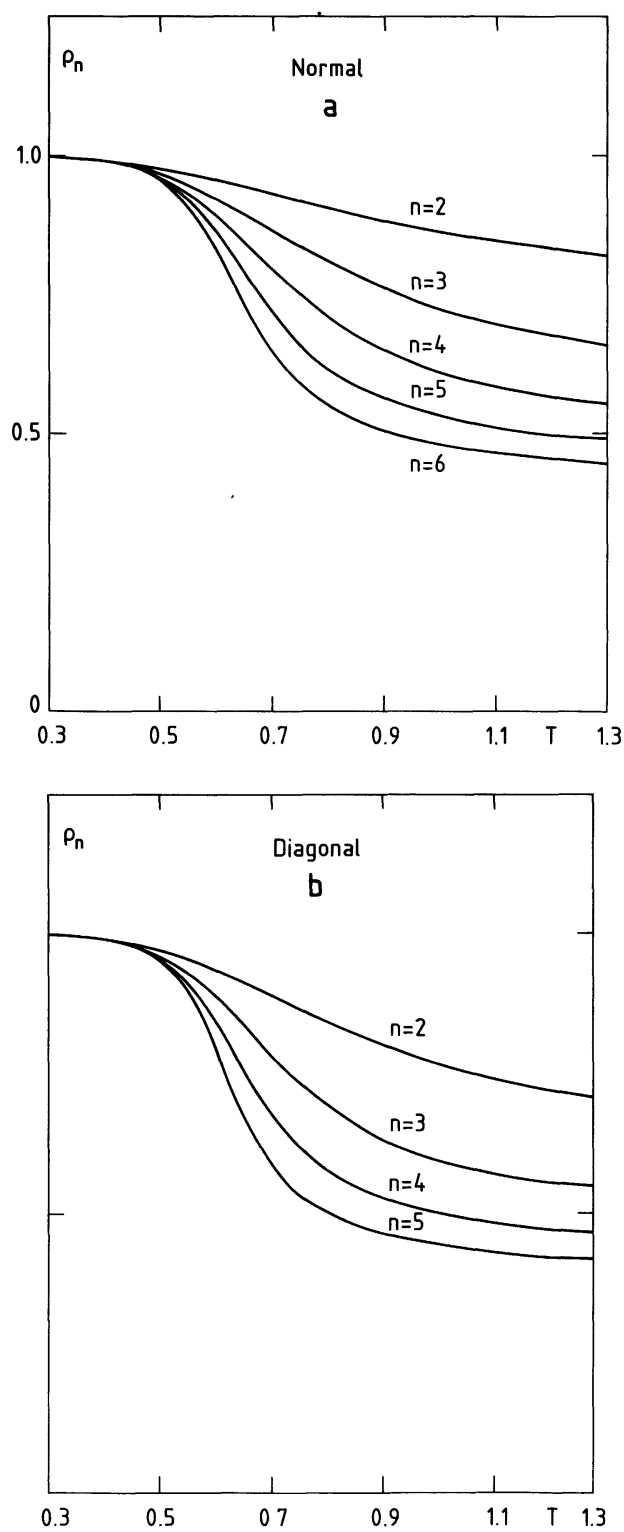

Fig. 3. - Density $\rho_{n}$ against temperature for different strip widths $n:(a)$ normal strip direction; $(b)$ diagonal strip direction. 
Another interesting quantity is the thermal expan$\operatorname{sion} \alpha_{n}$ that we define by

$$
\alpha_{n}=-\frac{1}{\rho_{n}} \frac{\mathrm{d} \rho_{n}}{\mathrm{~d} T}
$$

(see Fig. 4).

Let us now find the thermodynamic quantities in a case of a weak gel.

As explained in the introduction, equation 9, $g_{0 R}(x, T)$ also gives the grand canonical potential of a weak gel. Since on a strip we have

$$
g_{0 R} \sim[\lambda(x, T)]^{R} \quad \text { for large } R
$$

the grand canonical potential $\psi$ for a volume $n \times R$ is given by

$$
\psi=-T R \log [\lambda(x, T)]
$$

This means that, for a weak gel, for a given choice of $x$ and the temperature $T$ the pressure is given by:

$$
p=\frac{T}{n} \log [\lambda(x, T)] .
$$
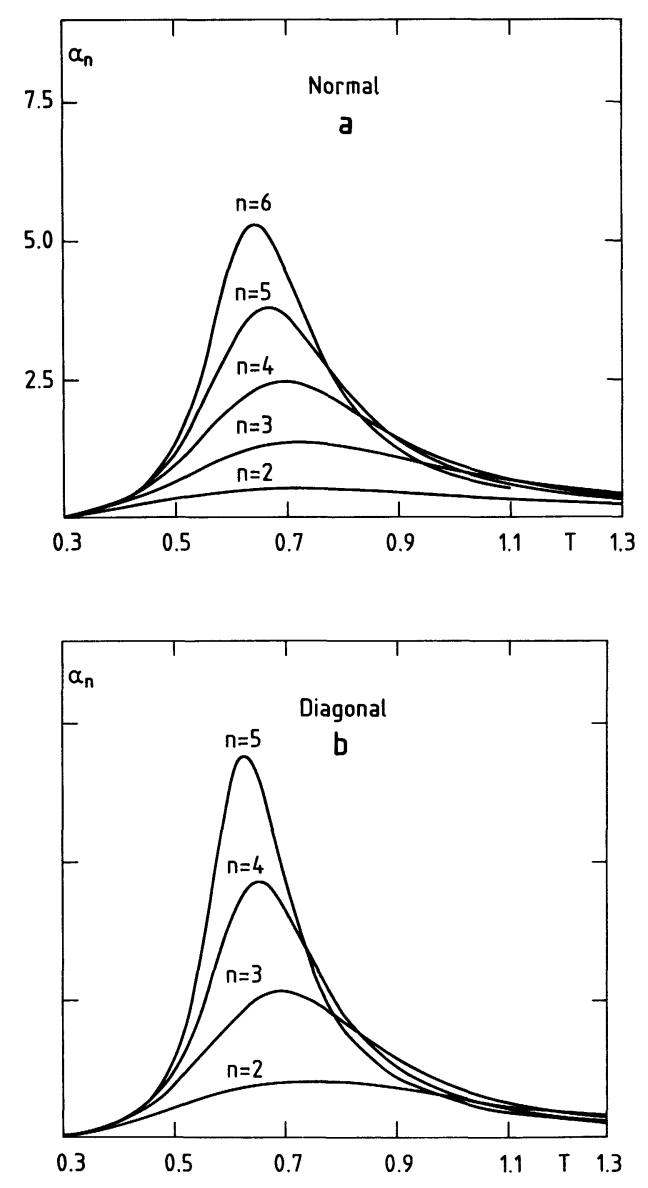

Fig. 4. - Thermal expansion $\alpha_{n}$ against temperature for different strip widths $n:(a)$ normal strip direction; $(b)$ diagonal strip direction.
Then the free energy $f_{\text {gel }}$ per atom in the gel is given by

$$
f_{\mathrm{gel}}=T\left[\log x-\left(\frac{\partial \log \lambda}{\partial \log x}\right)^{-1} \log \lambda\right]
$$

and the density $\rho_{\text {gel }}$ is given by

$$
\rho_{\mathrm{gel}}=\frac{1}{n} \frac{\partial \log \lambda}{\partial \log x} .
$$

One should note that in the case of a gel the whole $x-T$ plane is accessible, even the region where the pressure $p$ is negative which corresponds to a force that swells the gel. One can also note that the condition (16) which gave us the properties of an animal in the limit $N \rightarrow \infty$ can be seen here as the condition that the pressure vanishes. This is not surprising because when we consider an animal, we do not restrict it to a given volume and therefore $p=0$. In the case of weak gels, one can of course calculate everything from equations 25 and 26 and distinguish specific heats at constant pressure or constant volume.

From (26) one can calculate the compressibility $\kappa_{n}$ defined by

$$
\kappa_{n}=\frac{1}{\rho_{n}} \frac{\partial \rho_{n}}{\partial p} .
$$

In figure 5 we represent the compressibility as a function of temperature for several strip widths at $p=0$.

From figures 2 to 5 one can see strong evidence for a phase transition between a good solvent phase (at high temperatures) and a poor solvent phase (at low temperatures).

In figures 2,4 and 5 , clearly a singularity is built up with'increasing strip width $n$ and the two dimensional situation is apparently approached in a systematic way. The approach seems to be more rapid for the diagonal case but in means of numerical effort both cases are about the same because one point of the normal strip for $n=5$ took about the same computer time as one point of the diagonal strip for $n=4$. (See the table in the appendix.)

\section{Two-strip renormalization, critical line.}

In this section we will use usual phenomenological renormalization [34, 35] to obtain the critical line $\tilde{x}(T)$. If we consider our model at a fixed temperature $T$ and vary the fugacity $x$ we will have a transition at $\tilde{x}(T)$ where the correlation length $\xi(x, T)$ diverges.

For $T=\infty$ the transition is identical to that of usual lattice animals [28] and is thus of second order with an exponent $v \approx 0.64$ for the correlation length. At low temperatures one expects $v$ to be equal $1 / d=$ $1 / 2$.

We first make the usual assumption of the phenomenological renormalization that

$$
\frac{1}{n} \xi_{n}(\tilde{x}(T), T)=\frac{1}{m} \xi_{m}(\tilde{x}(T), T)
$$



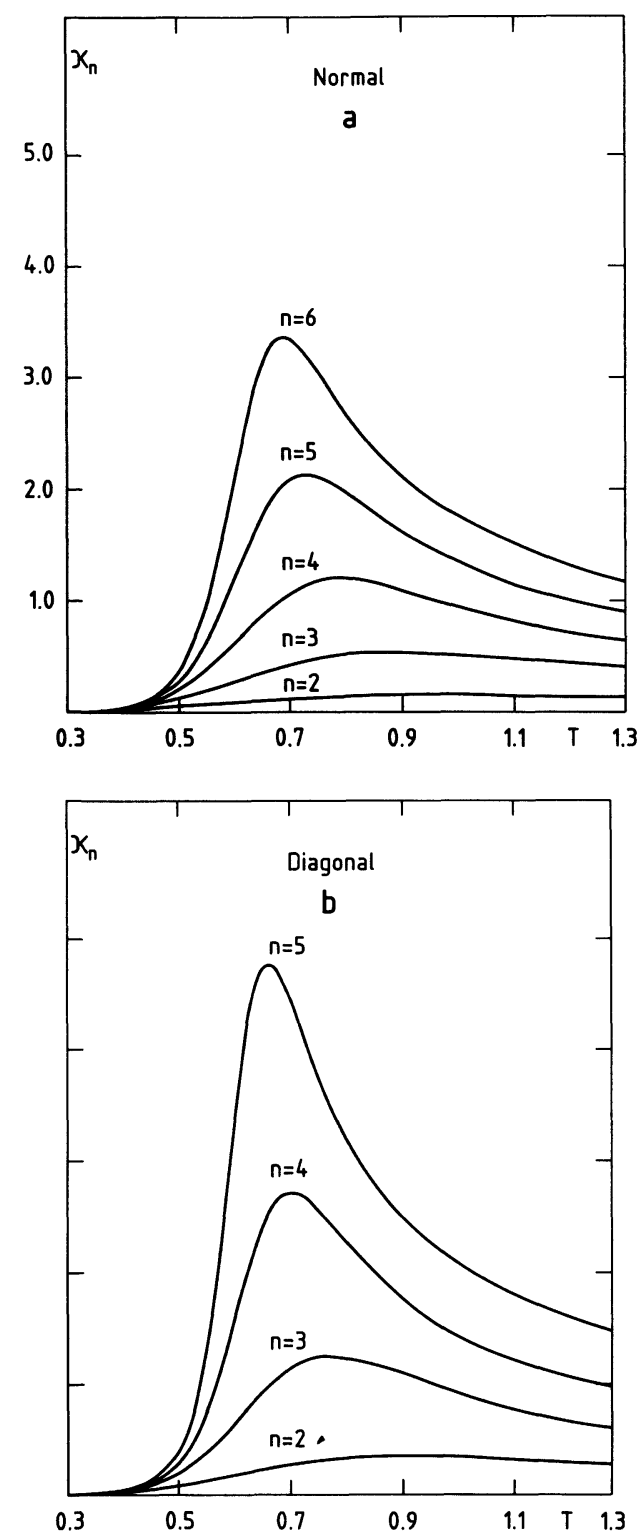

Fig. 5. - Compressibility $\kappa_{n}$ against temperature for different strip widths $n:(a)$ normal strip direction; (b) diagonal strip direction.

holds for the correlation length $\xi_{n}$ of the strip of width $n$ [28] defined by (14). Applying (28) to two strips of width $n$ and $n-1$ and fixed $T$ we obtain for each $n$ an estimate for the critical line shown in figure 6 for the normal and the diagonal case. One sees for increasing $n$ a good convergence at all temperatures. This convergence is extremely rapid at low temperatures.

With the two-strip renormalization one can also calculate the exponent $v$ by looking at the derivative $\xi^{\prime}$ of the correlation length with respect to $x[28]$ :

$$
v_{n}^{-1}=\frac{\log \left[\xi_{n}^{\prime}(\tilde{x}, T) / \xi_{n-1}^{\prime}(\tilde{x}, T)\right]}{\log [n /(n-1)]}-1 .
$$

This $v_{n}$ is presented in figure 7 as a function of temperature for the normal and the diagonal case. At low
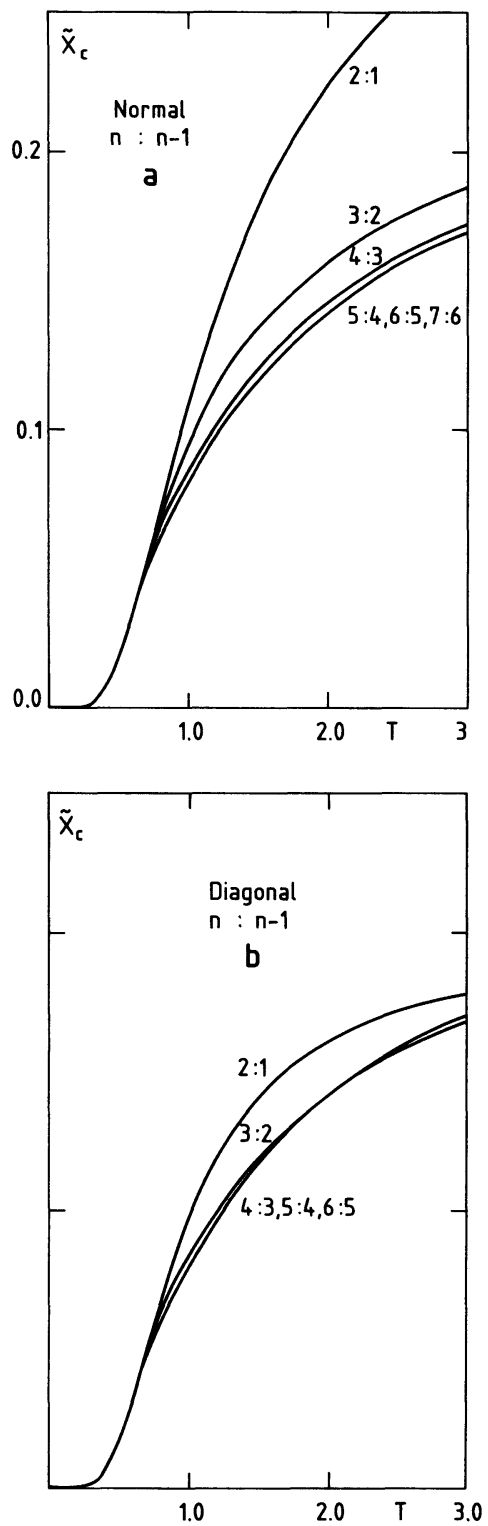

Fig. 6. - Value $\tilde{x}(T)$ at which the correlation length diverges against temperature obtained from a $n$ to $n-1$ renormalization for different pairs of values $n, n-1$. (a) Normal strip direction; $(b)$ diagonal strip direction. In the terminology of gels this is the $x$ - $T$ phase diagram. Note that for $n \geqslant 5$ in the normal case and for $n \geqslant 4$ in the diagonal case the lines are so close that they can not be distinguished in the plot.

temperatures one clearly obtains the exponent $1 / d$ and at high temperatures the lattice animal exponent is asymptotically approached for increasing $n$. One should note that for increasing $n, v$ is more and more constant in the high temperature phase and in the low temperature phase.

At about $T=0.535$ there seems to be a point where all curves cross with a value of the exponent of about $v \simeq 0.512$. On the high temperature side of $\theta$ the exponent increases sharply to a value which in the large $n$ limit might saturate to about $v \simeq 0.75$ before 

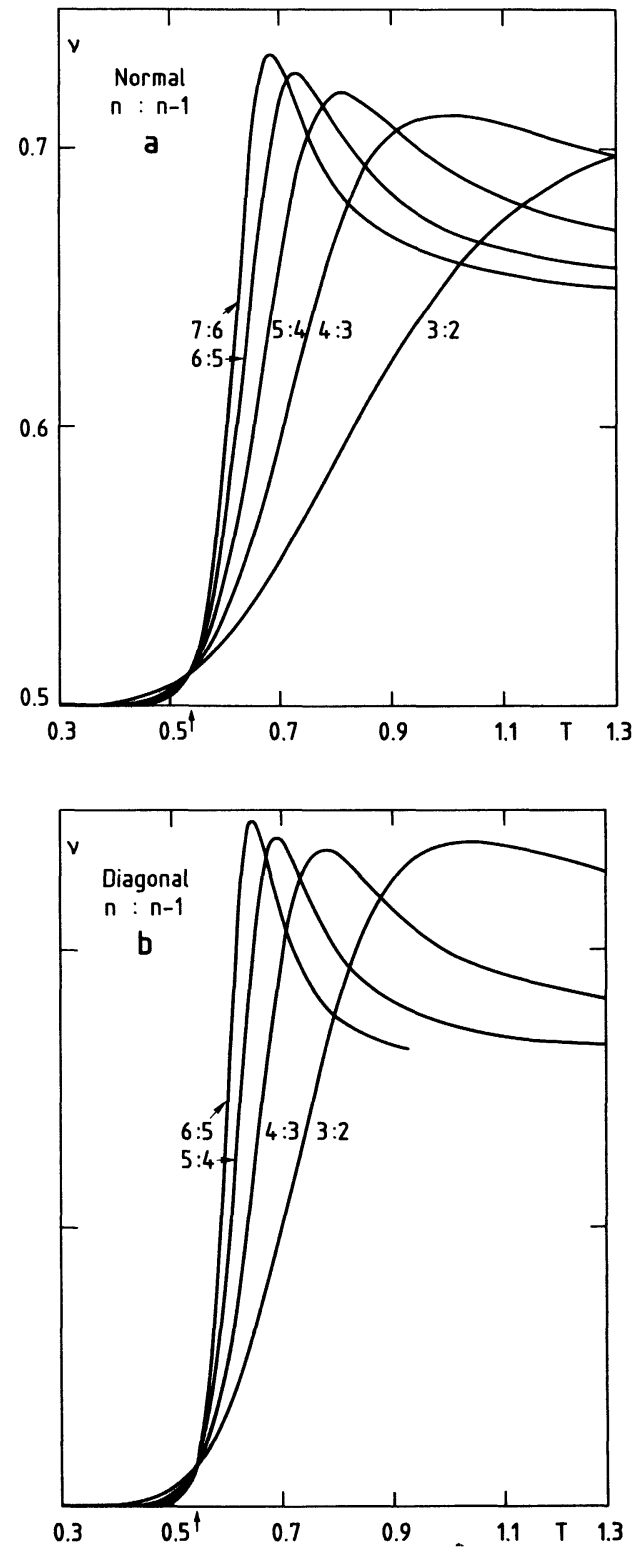

Fig. 7. - Exponent $v$ of the correlation length against temperature obtained from a $n$ to $n-1$ renormalization for different pairs of values $n, n-1:(a)$ normal strip direction; (b) diagonal strip direction. Note that all the lines cross in one point at a temperature of about $\theta=0.535$.

it goes down to the high temperature exponent $v \approx 0.64$. The strong change of $v$ around $\theta$ indicates that this is the theta region. We note that the value $v$ at which all the curves cross and the maximum value of $v$ are close to the two tricritical exponents that we will calculate in the next section.

In the language of the gel figure 6 represents the phase diagram between a dense phase at the left side and a swollen phase at the right side. The transition line (which is at the same time the isobar with $p=0$ ) has a first-order transition for low temperatures which changes to a second-order transition if one goes to high temperatures. This can be seen in figure 8 where the density is shown as a function of the fugacity $x$ at three different temperatures. For $T=0.25$ one already sees clearly a jump in the density for small $n$. At $T=1.0$ the change in density appears smooth also for larger $n$. It should be noted that at low temperatures the correlation length diverges on the line $\tilde{x}(T)$ although the transition is first order. This is not $a$ priori evident but one can indeed test that the transition points extrapolated from figure 8 to large $n$ also agree for low temperatures with the transition line obtained in figure 6.

\section{Determination of the theta point and its exponents.}

In this section we will present two different ways of calculating a tricritical point with phenomenological renormalization and apply them to find the collapse temperature $\theta$ of our model.

At a tricritical point we shall consider that the correlation length scales [36] as

$$
\xi_{n}(x, T) \sim n F\left(n^{\frac{i}{v_{1}}} u, n^{\frac{1}{v_{2}}} v\right)
$$

with two tricritical exponents $v_{1}$ and $v_{2}$, a scaling function $F$ and the scaling fields :

$$
\begin{aligned}
& u=a\left(x-x_{\mathfrak{t}}\right)+b(T-\theta)+\cdots \\
& v=c\left(x-x_{\mathfrak{t}}\right)+d(T-\theta)+\cdots .
\end{aligned}
$$

In order to determine the fixed point $(u, v)=(0,0)$ we need one more equation than in the critical case (Eq. 28).

In the first method that we present we use three different strip widths $n, m$ and $l$ and with the two equations of the type (28) :

$$
\frac{\xi_{n}\left(x_{\mathfrak{v}}, \theta\right)}{n}=\frac{\xi_{m}\left(x_{\mathfrak{t}}, \theta\right)}{m}=\frac{\xi_{l}\left(x_{\mathfrak{v}}, \theta\right)}{l}
$$

we obtain the tricritical values $x_{\mathrm{t}}$ and $\theta$. A three-width method of this kind was also used in directed problems [31, 32]. To calculate the two tricritical exponents one takes the derivatives of $\xi_{n}$

$$
\begin{aligned}
& \partial_{x} \xi_{n}=\frac{\partial \xi_{n}}{\partial x}=n\left(a n^{\frac{1}{v_{1}}} \frac{\partial F}{\partial u}+c n^{\frac{1}{v_{2}}} \frac{\partial F}{\partial v}\right) \\
& \partial_{T} \xi_{n}=\frac{\partial \xi_{n}}{\partial T}=n\left(b n^{\frac{1}{v_{1}}} \frac{\partial F}{\partial u}+d n^{\frac{1}{v_{2}}} \frac{\partial F}{\partial v}\right)
\end{aligned}
$$

for the three strip widths and by eliminating the directional constants one obtains that both exponents are solutions of an equation

$$
\frac{\partial_{x} \xi_{n}-\left(\frac{n}{m}\right)^{1+\frac{1}{v_{i}}} \partial_{x} \xi_{m}}{\partial_{x} \xi_{n}-\left(\frac{n}{l}\right)^{1+\frac{1}{v_{i}}} \partial_{x} \xi_{l}}=\frac{\partial_{T} \xi_{n}-\left(\frac{n}{m}\right)^{1+\frac{1}{v_{i}}} \partial_{T} \xi_{m}}{\partial_{T} \xi_{n}-\left(\frac{n}{l}\right)^{1+\frac{1}{v_{i}}} \partial_{T} \xi_{l}}
$$

The second method that we propose to localize the tricritical point is to use also the second largest 

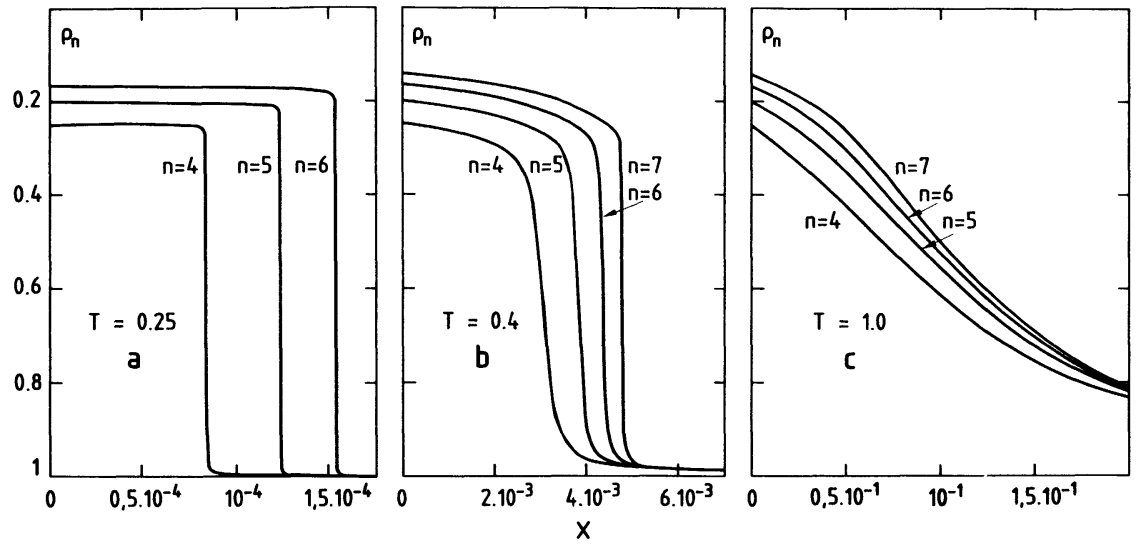

Fig. 8. - Density $\rho_{n}$ against the fugacity $x$ for different strip widths $n$ and different temperatures $T:(a) T=0.25,(b) T=$ 0.4 , (c) $T=1.0$. The strips are taken in normal direction.

eigenvalue $\lambda$ of the symmetrized transfer matrix (see the appendix).

With $\tilde{\lambda}$ we define a second correlation length

$$
\tilde{\xi}(x, T)=-\log ^{-1}[\lambda(x, T)]
$$

and use additionally to (28) the equation

$$
\frac{1}{n} \widetilde{\xi}_{n}\left(x_{t}, \theta\right)=\frac{1}{m} \widetilde{\xi}_{m}\left(x_{t}, \theta\right)
$$

to determine the two values $x_{t}$ and $\theta$. The exponents are in this case obtained by making for $\tilde{\xi}$ a scaling assumption of the type of (30) with the same scaling fields (31). Then taking the derivatives of $\xi$ and $\tilde{\xi}$ with respect to $x$ and $T$ for two strip widths and eliminating the directional constants one obtains that the exponents are the two solutions of

$$
q_{1}\left[\left(\frac{n}{m}\right)^{1+\frac{1}{v_{i}}}\right]^{2}-q_{2}\left(\frac{n}{m}\right)^{1+\frac{1}{v_{i}}}+q_{3}=0
$$

with

$$
\begin{aligned}
& q_{1}=\left(\partial_{T} \tilde{\xi}_{m}\right)\left(\partial_{x} \xi_{m}\right)-\left(\partial_{T} \xi_{m}\right)\left(\partial_{x} \tilde{\xi}_{m}\right) \\
& q_{2}=\left(\partial_{T} \tilde{\xi}_{n}\right)\left(\partial_{x} \xi_{m}\right)-\left(\partial_{T} \xi_{n}\right)\left(\partial_{x} \tilde{\xi}_{m}\right) \\
& \quad+\left(\partial_{T} \tilde{\xi}_{m}\right)\left(\partial_{x} \xi_{n}\right)-\left(\partial_{T} \xi_{m}\right)\left(\partial_{x} \tilde{\xi}_{n}\right) \\
& q_{3}=\left(\partial_{T} \tilde{\xi}_{n}\right)\left(\partial_{x} \xi_{n}\right)-\left(\partial_{T} \xi_{n}\right)\left(\partial_{x} \tilde{\xi}_{n}\right) .
\end{aligned}
$$

In figure 9 we plot the values we obtain for $\theta_{n}$ using strip widths $n, n-1$ and $n-2$ in (32) or $n$ and $n-1$ in (28) and (36). The data of $\theta_{n}$ are given in tables I and II. Arbitrarily we plot the data versus $1 / n$. We go up to $n=10$ for the normal case and up to $n=8$ for the diagonal case. In the normal case the data do not converge monotonically but all four curves seem to converge to the same value. We extract a value of $\theta$ :

$$
\theta=0.535 \pm 0.005 \text {. }
$$

Similar curves for the tricritical fugacity yield $x_{t}=$

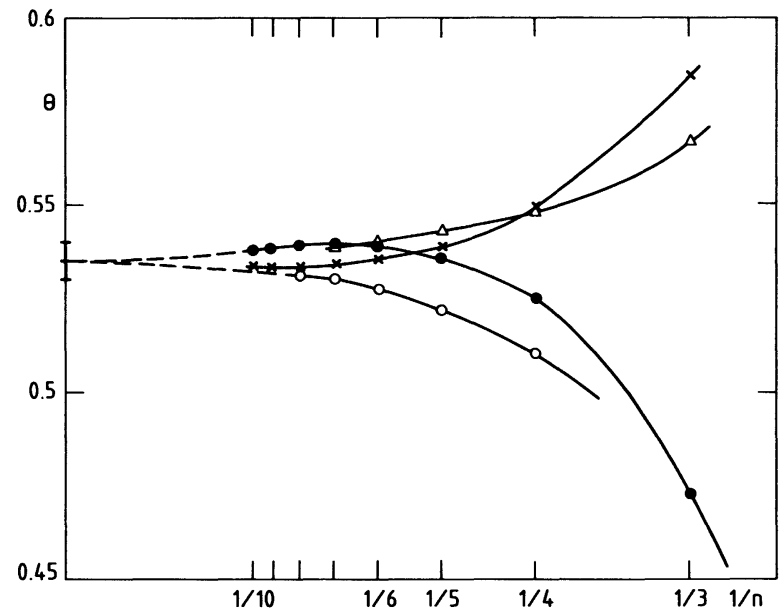

Fig. 9. - Tricritical temperature $\theta$ plotted against $n^{-1}$ where $n$ is the largest strip width used to obtain $\theta$. We show the values from a renormalization using three lengths $n$, $n-1$ and $n-2$ for the normal strip direction $(0)$ and the diagonal strip direction $(\Delta)$ and the values from a $n$ to $n-1$ renormalization using the two largest eigenvalues of the transfer matrix for the normal strip direction $(x)$ and the diagonal strip direction (O). Our prediction (39) is indicated on the vertical axis.

$0.0230 \pm 0.0004$. In tables I and II we show our results for the two tricritical exponents. In figure 10 we plot them versus $1 / n$. The plot for $v_{1}$ shows again some non-monotonic curves.

We extrapolate :

$$
v_{1}=0.5095 \pm 0.0030
$$

and

$$
v_{2}=0.775 \pm 0.025 \text {. }
$$

Thus the crossover exponent is

$$
\phi=\frac{v_{1}}{v_{2}}=0.657 \pm 0.025 \text {. }
$$


Table I. - Values for $\theta, v_{1}$ and $v_{2}$ obtained from a renormalization using three different strip widths.

\begin{tabular}{|c|c|c|c|c|c|c|c|c|}
\hline \multirow{2}{*}{\multicolumn{3}{|c|}{ Widths }} & \multicolumn{2}{|c|}{$\theta$} & \multicolumn{2}{|c|}{$v_{1}$} & \multicolumn{2}{|c|}{$v_{2}$} \\
\hline & & & normal & diagonal & normal & diagonal & normal & diagonal \\
\hline 3 & 2 & 1 & 0.47291 & 0.57615 & 0.5030 & 0.5296 & 1.2094 & 0.8436 \\
\hline 4 & 3 & 2 & 0.52476 & 0.54923 & 0.5073 & 0.5183 & 0.9575 & 0.8222 \\
\hline 5 & 4 & 3 & 0.53567 & 0.54326 & 0.5101 & 0.5156 & 0.8891 & 0.8154 \\
\hline 6 & 5 & 4 & 0.53866 & 0.54068 & 0.5116 & 0.5143 & 0.8559 & 0.8114 \\
\hline 7 & 6 & 5 & 0.53920 & 0.53926 & 0.5123 & 0.5135 & 0.8375 & 0.8087 \\
\hline 8 & 7 & 6 & 0.53899 & 0.53838 & 0.5125 & 0.5129 & 0.8268 & 0.8068 \\
\hline 9 & 8 & 7 & 0.53860 & & 0.5124 & & 0.8203 & \\
\hline 10 & 9 & 8 & 0.53820 & & 0.5123 & & 0.8158 & \\
\hline
\end{tabular}

Table II. - Values for $\theta, v_{1}$ and $v_{2}$ obtained from a $n$ to $n-1$ renormalization and using two eigenvalues of the transfer matrix.

\begin{tabular}{|c|c|c|c|c|c|c|c|}
\hline \multirow{2}{*}{\multicolumn{2}{|c|}{ Widths }} & \multicolumn{2}{|c|}{$\theta$} & \multicolumn{2}{|c|}{$v_{1}$} & \multicolumn{2}{|c|}{$v_{2}$} \\
\hline & & normal & diagonal & normal & diagonal & normal & diagonal \\
\hline 3 & 2 & 0.58422 & & 0.3418 & & 0.5278 & \\
\hline 4 & 3 & 0.54923 & 0.50992 & 0.5150 & 0.5069 & 0.6490 & 0.7486 \\
\hline 5 & 4 & 0.53861 & 0.52235 & 0.5128 & 0.5078 & 0.6975 & 0.7468 \\
\hline 6 & 5 & 0.53512 & 0.52742 & 0.5114 & 0.5080 & 0.7163 & 0.7483 \\
\hline 7 & 6 & 0.53389 & 0.52994 & 0.5106 & 0.5081 & 0.7266 & 0.7502 \\
\hline 8 & 7 & 0.53348 & 0.53137 & 0.5100 & 0.5081 & 0.7332 & 0.7521 \\
\hline 9 & 8 & 0.53339 & & 0.5096 & & 0.7380 & \\
\hline 10 & 9 & 0.53343 & & 0.5093 & & 0.7416 & \\
\hline
\end{tabular}
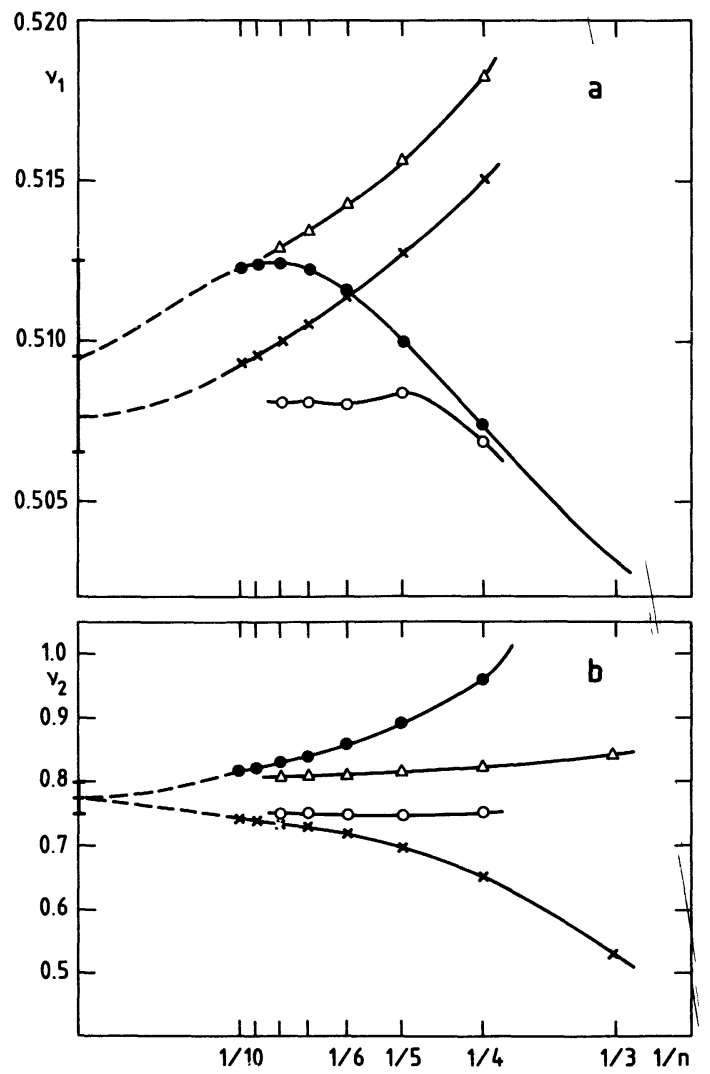

$\leftarrow$ Fig. 10. - Tricritical exponents $v_{1}$ (in $a$ ) and $v_{2}$ (in $b$ ) plotted against $n^{-1}$, where $n$ is the largest strip width used in a calculation. We show the values obtained by renormalizing with three lengths $n, n-1$ and $n-2$ for the normal $(\bullet)$ and the diagonal $(\Delta)$ strip direction. We also show the values obtained from a $n$ to $n-1$ renormalization using the two largest eigenvalues of the transfer matrix for the normal $(x)$ and the diagonal $(O)$ strip direction. Our predictions (40) and (41) are indicated on the vertical axis.

The exponent $v_{1}$ gives the size $\left\langle R^{2}\right\rangle$ of an animal of $N$ sites in the limit $N \rightarrow \infty$ at the temperature $\theta$

$$
\left\langle R^{2}\right\rangle \sim N^{2 v_{1}},
$$

whereas the exponent $v_{2}$ is the exponent of a thermal length which diverges like $(\theta-T)^{-v_{2}}$ and represents the correlations of the thermal fluctuations.

\section{Thermodynamic quantities at the theta point.}

After having located the tricritical point we can go back to the data presented in section 3 and analyse them in the vicinity of the tricritical point. We will only discuss here the finite size effects in the case of the density $\rho$. 
Finite size scaling tells us that, for large widths $n$, there exists a scaling function $H$ such that [10]

$$
\rho_{n} n^{2-\frac{1}{v_{1}}}=H\left[(T-\theta)\left(\rho_{n} n^{2}\right)^{\phi}\right]
$$

If we use the values of $\theta, v_{1}$ and $\phi$ that we have calculated in section 5 and plot the left-hand side of equation 43 against the argument of the function $H$ we get figure 11. We see that the points indeed lie more or less on one curve, the function $H$. One sees in figure 11 some systematic deviations from this curve due to the fact that $n$ is small in our case but we believe that (43) is valid in the asymptotic limit $n \rightarrow \infty$. Figure 11 confirms rather well the values of $\theta, v_{1}$ and $\phi$ obtained in the previous section. This was not possible in the Monte-Carlo calculations of reference 10 where the authors had to choose a very different $\phi$ to fit a curve to (43) in the case of linear chains.

In principle, the knowledge [37] of the exponents $v_{1}$ and $v_{2}$ obtained in equations 40 and 41 allows us to find the singular behaviour of quantities in the neighbourhood of the tricritical point. For example, the density $\rho$ of the two-dimensional system vanishes in the following way at $T=\theta$

$$
\rho(T) \sim(\theta-T)^{\left(2-\frac{1}{v_{1}}\right) v_{2}} .
$$

Thus the exponent of the density $\left(2-1 / v_{1}\right) v_{2}$ is of order of 0.03 or 0.04 . To obtain the exponent $\alpha$ of the specific heat $C$ per site in the animal, we can come back to the scaling (Eq. 30) of the correlation length.

This scaling relation implies that, near the tricritical point, the critical line $\tilde{x}(T)$ of the two-dimensional problem has the following singular part

$$
\left(\tilde{x}(T)-x_{t}\right)_{\text {sing. }} \sim|T-\theta|^{\frac{v_{2}}{v_{1}}}
$$

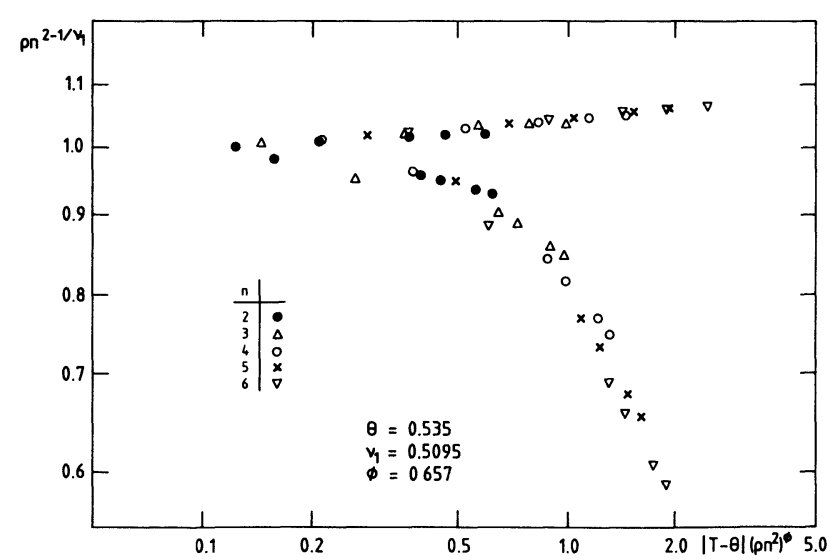

Fig. 11. - Finite size scaling plot for the density $\rho$. We plot $\rho . n^{2-1 / v_{1}}$ against $|T-\theta|\left(\rho n^{2}\right)^{\phi}$ for different $T$ and $n$ using $\theta=0.535, v_{1}=0.5095$ and $\phi=0.657$. The points lie on two curves the upper one is for $T<\theta$, the lower one for $T>\theta$.
As $\tilde{x}(T)$ gives the free energy per site (see Eq. 4), one sees that the exponent $\alpha$ is given by

$$
\alpha=2-\frac{v_{2}}{v_{1}} \simeq 0.48
$$

since one has

$C(T)=-T^{2} \frac{\mathrm{d} \tilde{x}}{\mathrm{~d} T^{2}}-2 T \frac{\mathrm{d} \tilde{x}}{\mathrm{~d} T} \sim|T-\theta|^{-\alpha}$

Relation 46 can be understood easily by writing

$$
\alpha=2-\bar{d} v_{2}
$$

where $\bar{d}=1 / v_{1}$ is the fractal dimension of the lattice animal at the $\theta$ point.

\section{Summary and conclusions.}

We have presented a model with two parameters, temperature and fugacity, and applied to it the transfer matrix technique on finite strips. This technique has proven to be very powerful in two dimensions. We have located the tricritical point and obtained its exponents in two different ways. Physical quantities like specific heat, thermal expansion and compressibility were calculated. Different possibilities to physically interprete the generating function were proposed.

For the collapse of a two-dimensional gel we have found a region of first-order transitions and a region of second-order transitions. The two regions are separated by a tricritical point, which we may call a theta point, with exponents $v_{1}=0.5095 \pm 0.0030$ and $\phi=0.657 \pm 0.025$. These exponents are not in agreement with recently found Flory-exponents $v_{1}=$ $7 / 12$ and $\phi=5 / 6[15]$. This is not surprising since the upper tricritical dimension for this problem is $d_{\mathrm{t}}=6$.

A three-dimensional calculation with the transfer matrix method is much more difficult because of the size of the transfer matrix. Therefore we encourage experiments on the two-dimensional collapse of a branched polymer to verify the qualitative features of the model and the critical and tricritical exponents.

We hope to make the same calculations for the collapse transition of a linear polymer in $d=2$. Our preliminary results show that there is an odd-even imparity in the strip width $n$. However, we hope that the methods used in section 5 will give accurate estimates of the exponents, also for linear polymers.

\section{Acknowledgments.}

We would like to thank J. L. Lebowitz for his encouragements to work on collapse transitions. We also want to thank R. B. Griffiths and R. B. Pearson for illuminating discussions and B. Duplantier for a critical reading of the manuscript. 


\section{Appendix}

\section{CAlculation of THE TRANSFer MatriX.}

a) Set of configurations of a column. - As we want to study the lattice animal of the $n \times \infty$ strip by looking only at one column to which the transfer matrix is applied, the first step in the calculation of the transfer matrix is to determine the set of possible configurations of a column for each $n$.

First we note that each site of the column can be occupied or empty and only the configuration with all sites empty is not allowed because it would destroy the end-to-end connectedness of the animal. In this way we would have $2^{n}-1$ configurations. But the column that one considers must contain all the information on the columns at its left, namely if two sites in the column are separated they can nevertheless be connected through these other columns or not. As an example we show the configurations for $n=4$ in figure 12 and denote already connected sites (i.e. occupied sites which are connected to the left part of the strip) by the same symbol and not connected sites (i.e. occupied sites which are not connected to the left part of the strip) by different symbols.

Finally, as can also be seen in figure 12, the number of configurations can be heavily reduced if one uses the spatial symmetries of the system namely the reflexion symmetry around the axis along the strip and in the case of periodic boundaries which we consider the rotational symmetry. To get all the $s$ different possible configurations $(s=6$ for $n=4)$ is, after all these considerations, not a trivial task.

We obtain all the configurations by the following algorithm with the computer :

1. begin with a configuration which is for sure present, e.g. all sites occupied ;

2. put on this configuration $C_{1}$ all possible $2^{n}-1$ occupied-empty configurations $F_{i}$ and determine their connectivity properties due to the fact that $F_{i}$ follows $C_{1}$, i.e. determine in figure 12 how to put the symbols $(e, x$, etc.). Configurations that would leave an isolated cluster behind or destroy the connection to infinity are thrown away;

3. symmetrize the $F_{i}$ (taking into account the connectivity properties). For this symmetrization one must define in a unique way which of two configura-

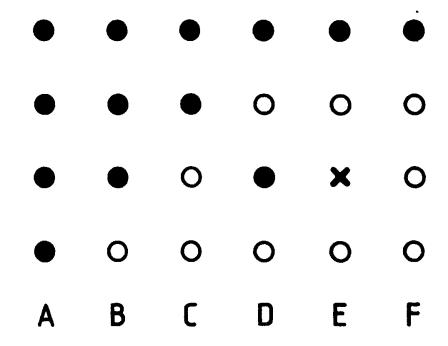

Fig. 12. - The six different configurations that can occur in a strip of width $n=4$. Occupied and connected site : 0 , occupied and not connected site : $\times$, empty site $: 0$.

tions identical by a symmetry one prefers for a final description of the set of configurations as that of figure 12. Many equally effective definitions are possible;

4. look if the final result of the symmetrization procedure is already one of the configurations $C_{j}$; if not, define it as a new element in the set $\left\{C_{j}\right\}$;

5. repeat $2 .-4$. by putting the $2^{n}-1$ configurations $F_{i}$ on $C_{j}$ for $j>1$ until one has done it for all the $C_{j}$ that one has created.

One sees that the above algorithm automatically is exhausted if one has found all the $s$ configurations $C_{j}$.

b) Construction of the transfer matrix. - The algorithm presented for the construction of all the configurations of a column has the advantage that one can with it simultaneously construct the transfer matrix. One considers in step 2 of the algorithm the $F_{i}$ that one puts on the $C_{j}$ to be the configurations of the $(R+1)$ th column put on the $R$ th column and one calculates the contribution this has for the transfer matrix. These contributions to each matrix element are summed up.

Figure 12 gives the only six configurations $A, B, C$, $D, E$ and $F$ which can occur on a strip of width 4 . For configuration $A$ of figure 12 let us denote by $A_{R}$ the $g_{0 R}(A)$ defined in (10) of section 2. $A_{R}$ is therefore the generating function of all the animals which realize configuration $A$ at column $R . B_{R}, C_{R}, \ldots, F_{R}$ are defined analogously for configurations $B, C, \ldots, F$ of figure 12. The recurrence relations between $A_{R}$, $B_{R}, \ldots, F_{R}$ and $A_{R+1}, B_{R+1}, \ldots, F_{R+1}$ are :

$$
\begin{aligned}
& A_{R+1}=x^{4} y^{5}\left[y^{3} A_{R}+y^{2} B_{R}+y C_{R}+y D_{R}+y E_{R}+F_{R}\right] \\
& B_{R+1}=x^{3} y^{2}\left[4 y^{3} A_{R}+\left(y^{3}+3 y^{2}\right) B_{R}+2\left(y^{2}+y\right) C_{R}+2\left(y^{2}+y\right) D_{R}+2 y^{2} E_{R}+3 y F_{R}\right] \\
& C_{R+1}=x^{2} y\left[4 y^{2} A_{R}+2\left(y^{2}+y\right) B_{R}+\left(y^{2}+2 y\right) C_{R}+4 y D_{R}+2 y F_{R}\right] \\
& D_{R+1}=x^{2} y^{2}\left[2 A_{R}+B_{R}+D_{R}\right] \\
& E_{R+1}=x^{2} y\left[B_{R}+2 C_{R}+y E_{R}+F_{R}\right] \\
& F_{R+1}=x y\left[4 A_{R}+3 B_{R}+2 C_{R}+2 D_{R}+F_{R}\right] .
\end{aligned}
$$


Table A.I. - The sizes of the transfer matrices for normal strips and diagonal strips as a function of the strip width $n$.

\begin{tabular}{|c|c|c|}
\hline$n$ & Normal strip & Diagonal strip \\
\hline 2 & 2 & 2 \\
3 & 3 & 5 \\
4 & 6 & 13 \\
5 & 9 & 28 \\
6 & 19 & 75 \\
7 & 31 & 190 \\
8 & 67 & 562 \\
9 & 129 & \\
10 & 290 & \\
\hline
\end{tabular}

Note that for instance configuration $F$ on $E$ gives no contribution because otherwise the unconnected site of $E$ would remain unconnected. Details like this must be taken into account in step 2 of the algorithm.

We see that, after we have gone through the algorithm, the transfer matrix is constructed.

c) Sizes of transfer matrices. - The main numerical limitation with the transfer matrix method is that the size of the matrix increases rapidly with the width $n$ of the strip. In table A.I, we give the sizes $s$ of the transfer matrices once we have used all the symmetries.

Note added in proof: A. Coniglio has obtained in the context of the Potts Model results on a model similar to ours. By a Migdal Kadanoff renormalization which usually does not give very accurate exponents, he finds a value of $v_{1}$ very close to ours but a much higher value of $v_{2}$.

\section{References}

[1] FloRy, P. J., Principles of Polymer Chemistry (Cornell University Press, Ithaca) 1953.

[2] De Gennes, P. G., J. Physique-Lett. 36 (1975) L55; 39 (1978) L299.

[3] Domb, C., Polymer 15 (1974) 259.

[4] Fisher, M. E. and Hiley, B. J., J. Chem. Phys. 34 (1961) 1253.

[5] Rapaport, D. C., J. Phys. A 10 (1977) 637.

[6] SANCHEZ, I. C., Macromolecules 12 (1979) 980.

[7] Webman, I., Lebowitz, J. L. and Kalos, M. H., Macromolecules 14 (1981) 1495.

[8] Tobochnik, J., Webman, I., Lebowitz, J. L. and Kalos, M. H., Macromolecules 15 (1982) 549.

[9] Baumgärtner, A., J. Physique 43 (1982) 1407.

[10] Kremer, K., BaUmgärtner, A. and Binder, K., J. Phys. A 15 (1982) 2879.

[11] Nishio, I. and Sun, S. T., Swislow, G. and Tanaka, T., Nature 281 (1979) 208.

[12] Nierlich, M., Cotton, J. P. and Farnoux, B., J. Chem. Phys. 69 (1978) 1379.

[13] Takahaski, A., Yoshida, A. and Kawaguchi, M., Macromolecules 15 (1982) 1196.

[14] Vilanove, R., Rondelez, F., Phys. Rev. Lett. 45 (1980) 1502.

[15] Daoud, M., Pincus, P., Stockmayer, W. H., Witten, T., to appear in Macromolecules.

[16] Moore, M. A., J. Phys. A 10 (1977) 305.

[17] Duplantier, B., J. Physique 43 (1982) 991.

[18] Daoud, M. and Jannink, G., J. Physique 36 (1976) 281.

[19] Stauffer, D., Phys. Rep. 54 (1979) 1.

[20] Tanaka, T., Swislow, G. and Ohmine, I., Phys. Rev. Lett. 42 (1979) 1556 ;

Hochberg, A., Tanaka, T. and Nicoli, D., Phys. Rev. Lett. 43 (1979) 217;
Tanaka, T., Fillmore, D., Sun, S. T., Nishio, I., Swislow, G. and Shah, A., Phys. Rev. Lett. 45 (1980) 1636.

[21] Tanaka, K., Sci. American, Jan. 1981, p. 124.

[22] Khokhlov, A. R., Polymer 21 (1980) 376.

[23] Coniglio, A., Stanley, H. E. and Klein, W., Phys. Rev. B 25 (1982) 6805.

[24] Isaacson, J., Lubensky, T. C., J. Physique 42 (1981) 175.

[25] Lubensky, T. C., Isaacson, J., Phys. Rev. A 20 (1979) 2130.

[26] Stockmayer, W. H., Makromol. Chem. 35 (1960) 54.

[27] TANAKA, T., private communication.

[28] Derrida, B., De Seze, L., J. Physique 43 (1982) 475.

[29] Derrida, B. and Vannimenus, J., J. Physique Lett. 41 (1980) L473.

[30] Derrida, B., J. Phys. A 14 (1981) L5.

[31] Kinzel, W. and Yeomans, J. M., J. Phys. A 14 (1981) L163.

[32] Nadal, J. P., Derrida, B. and Vannimenus, J., J. Physique 43 (1982) 1561.

[33] Klein, D. J., J. Stat. Phys. 23 (1980) 561.

[34] Nightingale, M. P., Physica A 83 (1976) 561.

[35] Nightingale, M. P., J. Appl. Phys. 53 (1982) 7927, and references therein.

[36] Dos Santos, R. R. and Stinchcombe, R. B., J. P.hys. A 14 (1981) 2741.

[37] Griffiths, R. B., Phys. Rev. B 7 (1973) 545.

[38] Stephen, M. J., Phys. Lett. A 53 (1975) 363.

[39] Stauffer, D., Coniglio, A. and Adam, M., $A d v$ : Polymer Sci. 44 (1982) 103.

[40] Coniglio, A., J. Phys. A 16 (1983) L-187. 